\title{
Synthesis and Gas-Sensing Property of Highly Self-assembled Tungsten Oxide Nanosheets
}

\author{
Liangbin Hu, Pengfei Hu, Yong Chen*, Zehui Lin and Changjun Qiu* \\ School of Mechanical Engineering, University of South China, Hengyang, China
}

We report the synthesis of tungsten oxide $\left(\mathrm{WO}_{3}\right)$ nanosheets using a simple yet efficient hydrothermal technique free of surfactantand template. The $\mathrm{WO}_{3}$ nano-sheets are self-assembled as well to form ordered one-dimensional chain nanostructure. A comprehensive microscopic characterization reveals that the nano-sheets have triangular and circular two different shape edges, dislocation and stacking faults are also observed, which should have implications for our understanding of catalytic activity of ceria. We also propose a growth mechanism for the nano-sheets. As a result of this unique morphology, this $\mathrm{WO}_{3}$ nano-sheets are found to show excellent gas-sensing properties which can use as promising sensor materials detecting ethanol with low concentration.

OPEN ACCESS

Edited by:

Zhongchang Wang,

Laboratório Ibérico Internacional de Nanotecnologia (INL), Portugal

Reviewed by:

Jun Wang,

Shenzhen University, China

Xin Wang,

Shenzhen University, China

${ }^{*}$ Correspondence:

Yong Chen

chenyongjsnt@163.com

Changjun Qiu

qiuchangjun@hotmail.com

Specialty section:

This article was submitted to

Nanoscience,

a section of the journal

Frontiers in Chemistry

Received: 02 June 2018 Accepted: 12 September 2018

Published: 05 October 2018

Citation:

Hu L, Hu P, Chen Y, Lin Z and Qiu C (2018) Synthesis and Gas-Sensing Property of Highly Self-assembled

Tungsten Oxide Nanosheets.

Front. Chem. 6:452.

doi: 10.3389/fchem.2018.00452
Keywords: crystal structure, defects, nanosheets, highly self-assembled, gas-sensitivity

\section{INTRODUCTION}

Tungsten trioxide $\mathrm{WO}_{3}$ nanomaterials are extensively applied in electrochromic device, gas sensor and photocatalysts filed (Hai et al., 2016; Zhang et al., 2018). Great effort has been devoted to the control the specific size and shape of $\mathrm{WO}_{3}$ nanoparticles which can significantly impact properties of materials. Up to now, the $\mathrm{WO}_{3}$ nanoparticles with avariety of morphologies (such as nanowires, nano-rods, nano-plate, nano-spheres and so on) have been syhthesized successfully via highintensity ultrasound, rapid microwave, hydrothermal synthesis and other methods (Chai et al., 2016, Hu et al., 2017a, Xu et al., 2017; Chen et al., 2018; Parthibavarman et al., 2018; Zhan et al., 2018). Among all of those applied methods, hydrothermal synthesis technique has been concerned due to the merits of simple operation, low energy consumption, the possibility for large-scale industrialization and so on.

Here, we report the $\mathrm{WO}_{3}$ nano-sheets with highly self-assembled architecture synthesized via the hydrothermal synthesis process. Defects are observed in the nano-sheets, which may play a key role in affecting properties of $\mathrm{WO}_{3}$ nano-sheets. In addtion,we also test the response and selectivity of the sensor fabricated from the $\mathrm{WO}_{3}$ nano-sheets.

\section{MATERIALS AND METHODS}

All the reagents were analytical grade and without further purification. We adopted a facile hydrothermal method to synthesize the nanostructures. First of all, $32 \mathrm{ml}$ of deionized water and $8 \mathrm{ml}$ glycerol $\left(\mathrm{C}_{3} \mathrm{H}_{8} \mathrm{O}_{3}\right)$ were mixed into a mixture, then $1.6 \mathrm{nmol}$ of sodium tungstate dihydrate $\left(\mathrm{Na}_{2} \mathrm{WO}_{4} \cdot 2 \mathrm{H}_{2} \mathrm{O}\right)$ and $3 \mathrm{nmol}$ oleic acid $\left(\mathrm{C}_{18} \mathrm{H}_{34} \mathrm{O}_{2}\right)$ were dispersed into the mixture, and stirred for $15 \mathrm{~min}$ with a magnetic stirrer. Secondly, the $\mathrm{pH}$ of the mixture was adjusted to 1.25 by $\mathrm{HCl}$. After stirring for $15 \mathrm{~min}$, the solution was transferred into the Teflonlined stainless steel autoclave and treated at $120^{\circ} \mathrm{C}$ during $12 \mathrm{~h}$ under autogenously pressure. 


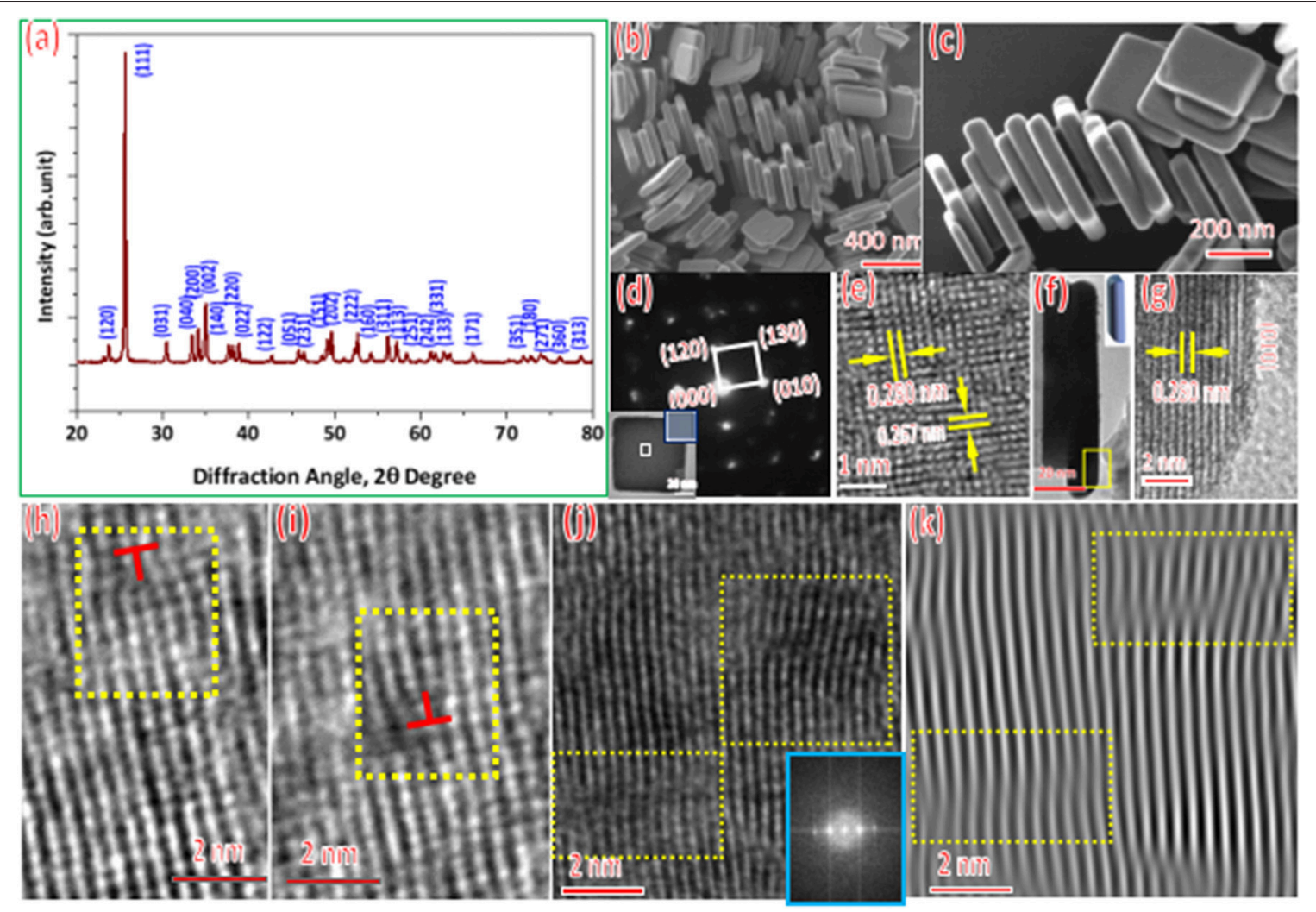

FIGURE 1 | Characterization of the samples prepared by hydrothermal method:(a) XRD spectrum, (b-c) SEM images, (d-g)TEM images, insert of (d) shows the TEM image of a WO3 nanoparticle, insert of (f) shows the model of the side of the nanosheet, (h-k) defects of samples, insert of (j) shows the Fast Fourier transform of the HRTEM image.

Finally, the obtained particles were washed by deionized and alcohol to remove the unexpected ions by high-speed centrifugation and then dried at $60^{\circ} \mathrm{C}$ for $10 \mathrm{~h}$ in air.

The characterization of speciemen as our previous work (Chen et al., 2013, 2014; Hu et al., 2017b). The process of measuring the gas sensitivity of the prepared nanomaterials is described in the literature (Guo and Wang, 2016). Response of the sensors was defined as the ratio of $\mathrm{Ra}$ (resistances in air) to $\mathrm{Rg}$ (resistances in target gases).

\section{RESULTS}

Figure 1a shows a typical XRD spectrum of the products, the diffraction peaks match well with those of a standard $\mathrm{WO}_{3} \cdot \mathrm{H}_{2} \mathrm{O}$ with orthorhombic structure (JCPDS No. 84-0886). The $\mathrm{WO}_{3} \cdot \mathrm{H}_{2} \mathrm{O}$ nano-particles exhibit rectangle shape with an average size of $\sim 400 \mathrm{~nm}$ and thickness of $30 \mathrm{~nm}$ (Figure $\mathbf{1 b}$ ). In addtion, one-dimensional chain nanostructure is self-assembled by the quadrilateral faces on the both sides of the nano-sheets. The face of nano-sheet are flat (Figures $\mathbf{1 b}, \mathbf{c}$ ). The corresponding SADP identifies that the structure of $\mathrm{WO}_{3}$ nano-sheets is orthorhombic (Figures 1d). Figure 1e shows a high-resolution TEM (HRTEM) image taken around the corner of a nanosheet. We determined the lattice spacing of the perpendicular lattice fringes to be $\sim 0.280$ and $\sim 0.267 \mathrm{~nm}$ which are belong to the $\mathrm{WO}_{3}(010)$ and (120) planes respectively. Figure 1g shows a HRTEM image taken from the corner area of the nanosheet Figure 1f from which lattice spacing is determined to be $\sim 0.280 \mathrm{~nm}$, in line with the distance between $\{010\}$ planes of $\mathrm{WO}_{3}$. In addtion, the edges of the nano-sheets are in an arc shape (insert of Figure 1f). Two different direction edge dislocations: positive edge dislocation (Figure $\mathbf{1 h}$ ) and negative edge dislocation (Figure 1i) are deteced by the HR-TEM, which should be critical for the properties of $\mathrm{WO}_{3}$. Apart from the defects on the surface, we also observed the stacking faults in nano-sheet (Figure 1j), which should impact the properties of $\mathrm{WO}_{3}$ nanoparticles as well. The stacking fault was observed obviously by the one-dimensionally filtered HR-TEM images of the $\mathrm{WO}_{3}$ nano-sheet (Figure 1k). Those dislocations and stacking faults may affect the catalytic activity or other properties of nano material (Wang et al., 2011, 2014, 2016; Sun et al., 2015).

Figure 2 shows the gases $\left(\mathrm{NH}_{3}, \mathrm{CH}_{3} \mathrm{OH}, \mathrm{C}_{6} \mathrm{H}_{6}\right)$ response of the sensor based on $\mathrm{WO}_{3}$ nanosheets. All of the gases were tested at an operating temperature of $300^{\circ} \mathrm{C}$ with a concentration of 30 ppm. In Figure 2, the results indicate that the sensor exhibited little responses to $\mathrm{NH}_{3}, \mathrm{C}_{6} \mathrm{H}_{6}$, to indicated that it was insensitive to $\mathrm{NH}_{3}, \mathrm{C}_{6} \mathrm{H}_{6}$. For ethanol, the highest response of the sensor was 25.6, while the responses to $\mathrm{NH}_{3}$ and $\mathrm{C}_{6} \mathrm{H}_{6}$ were no $>1$. 

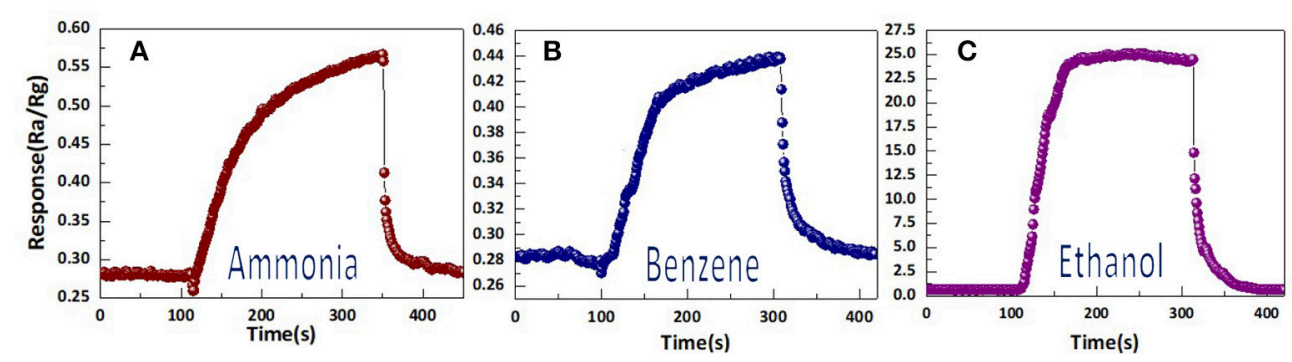

FIGURE 2 | The response of highly self-assembled tungsten oxide nanosheets sensor toward 30 ppm of different testing gases: (a) ammonia, (b) benzene, (c) ethanol.

\section{DISCUSSION}

In light of the aforementioned microstructural characterization, we propose a likely growth mechanism for the nanosheet. First, the $\mathrm{Na}_{2} \mathrm{WO}_{4} \cdot 2 \mathrm{H}_{2} \mathrm{O}$ is ionized to $\mathrm{WO}_{4}^{-}$. Then, the $\mathrm{WO}_{4}^{-}$ion react with $\mathrm{H}^{+}$which ionized by $\mathrm{HCl}$, forming the $\mathrm{H}_{2} \mathrm{WO}_{4}$ suspension. The $\mathrm{H}_{2} \mathrm{WO}_{4}$ suspension decomposes, at the high temperature and pressure during hydrothermal process, resulting in to the nucleation of $\mathrm{WO}_{3}$. The oleic acid acts as a soft template and controls the growth rate of different crystal plane owing to its selective absorption and desorption behavior. Then most of $\mathrm{WO}_{3}$ nano-sheets with $\{010\}$ exposure planes are selfassembled, forming one-dimensional chain nanostructures, due to the addition of oleic acid. The formation process can be described as follows:

$$
\begin{array}{r}
\mathrm{Na}_{2} \mathrm{WO}_{4} \cdot 2 \mathrm{H}_{2} \mathrm{O} \rightarrow 2 \mathrm{Na}^{+}+\mathrm{WO}_{4}^{-}+2 \mathrm{H}_{2} \mathrm{O} \\
\mathrm{HCl} \rightarrow \mathrm{H}^{+}+\mathrm{Cl}^{-} \\
2 \mathrm{H}^{+}+\mathrm{WO}_{4}^{-} \rightarrow \mathrm{H}_{2} \mathrm{WO}_{4} \\
\mathrm{H}_{2} \mathrm{WO}_{4} \rightarrow \mathrm{WO}_{3}+\mathrm{H}_{2}
\end{array}
$$

We imply that the $\mathrm{WO}_{3}$ nano-sheets can act as an efficient gas-sensing material for selective detection of ethanol. Such the sensing performance due to the fact that the diffusion of ethanol and its oxidation with $\mathrm{O}^{-}$or $\mathrm{O}^{2-}$ are very rapidly in nanoplates structures (Xiao et al., 2017). When sensor prepared by the $\mathrm{WO}_{3}$ nanosheets is exposed in air, the resistance of the $\mathrm{WO}_{3}$ nanosheets is increased by oxygen molecules which adsorbed on the surfaces of the $\mathrm{WO}_{3}$ nanosheets, trapping electrons in the conduction band and forming oxygen species $\left(\mathrm{O}^{-}, \mathrm{O}^{2-}\right)$. As ethanol is introduced to the sensor, the oxygen species $\left(\mathrm{O}^{-}\right.$, $\mathrm{O}^{2-}$ ) react with ethanol molecules on the surface of the $\mathrm{WO}_{3}$ nanosheets, which will release trapped electrons to conduction

\section{REFERENCES}

Ahmad, M. Z., Sadek, A. Z., Ou, J. Z., Yaacob, M. H., Latham, K., and Wlodarski, W. (2013). Facile synthesis of nanostructured $\mathrm{WO}_{3}$ thin films and their characterization for ethanol sensing. Mater. Chem. Phys. 141, 912-919. doi: 10.1016/j.matchemphys.2013. 06.022 band and resistance of the $\mathrm{WO}_{3}$ nanosheets is decreased (Ahmad et al., 2013).

\section{CONCLUSIONS}

We have adopted the hydrothermal technique to synthesize highly self-assembled $\mathrm{WO}_{3}$ nano-sheets using the tungsten resource $\mathrm{Na}_{2} \mathrm{WO}_{4} \cdot 2 \mathrm{H}_{2} \mathrm{O}$ and the soft template oleic acid. We demonstrated that the $\mathrm{WO}_{3}$ nano-sheets are mainly exposed with $\{010\}$ planes and crystal defects such as edge dislocations and stacking faults exist in single crystalline nano $\mathrm{WO}_{3}$ by microscopic investigations, which may be important for the catalytic activity of $\mathrm{WO}_{3}$. We indicate that the $\mathrm{WO}_{3}$ nano-sheets could be used as promising sensor material for detecting $\mathrm{CH}_{3} \mathrm{OH}$ with low concentration.

\section{AUTHOR CONTRIBUTIONS}

LH synthesized and characterized the microstructure of highly self-assembled tungsten oxide nanosheets and wrote the manuscript. $\mathrm{PH}$ and $\mathrm{ZL}$ tested the response and selectivity of the sensor fabricated from the $\mathrm{WO}_{3}$ nano-sheets. YC and CQ directed the experiment. All authors read and approved the manuscript.

\section{ACKNOWLEDGMENTS}

YC thanks the financial support by the Construct Program of the Key Discipline of Hunan province (Education Department of Hunan Province Bulletin grant no.[2014]85). ZL thanks the financial support by the Project of Research Study and Creativity Experiment Plan for College Students of the Hunan province (Education Department of Hunan Province Bulletin grant no.[2016]283).

Chai, Y., Ha, F. Y., Yam, F. K., and Hassan, Z. (2016). Fabrication of tungsten oxide nanostructure by sol-gel method. Proc. Chem. 19, 113-118. doi: 10.1016/j.proche.2016.03.123

Chen, G. C., Chua, X. F., Qiao, H. B., Ye, M. F., Chen, J., Gao, C., et al. (2018). Thickness controllable single-crystal $\mathrm{WO}_{3}$ nanosheets: highly selective sensor for triethylamine detection at room temperature. Mater. Lett. 226, 59-62. doi: 10.1016/j.matlet.2018.05.022 
Chen,Y., Liu, T. M., Chen, C. L., Guo, W. W., Sun, R., Lv, S., et al. (2013). Synthesis and characterization of $\mathrm{CeO}_{2}$ Nano-rods. Ceram. Int. 39, 6607-6610. doi: 10.1016/j.ceramint.2013.01.096

Chen,Y., Lv, S. H., Chen, C. L., Qiu, C. J., Fan, X. F., and Wang, Z. C. (2014). Controllable synthesis of ceria nanoparticles with uniform reactive $\{100\}$ exposure planes. J. Phys. Chem. C 118, 4437-4443. doi: 10.1021/ jp410625n

Guo, W. W., and Wang, Z. C. (2016). Composite of $\mathrm{ZnO}$ spheres and functionalized $\mathrm{SnO}_{2}$ nanofibers with an enhanced ethanol gas sensing properties. Mater. Lett. 169, 246-249. doi: 10.1016/j.matlet.2016.01.118

Hai, G., Huang, J., Jie, Y., Li, J., Cao, L., Zhang, G., et al. (2016). Influence of octadecylamine on the phase composition and the photocatalytic property of the tungsten oxide. Mater. Lett. 174, 134-137. doi: 10.1016/j.matlet.2016.03.049

Hu, P. F., Chen, Y., Chen, Y., Lin, Z. H., and Wang, Z. C., (2017a). Hydrothermal synthesis and photocatalytic properties of $\mathrm{WO}_{3}$ nanorods by using capping agent $\mathrm{SnCl}_{4} \cdot 5 \mathrm{H}_{2}$ O. Phys. E 92, 12-16. doi: 10.1016/j.physe.2017.05.004

Hu, P. F., Chen, Y., Sun, R., Chen, Y., Yin, Y. R., Wang, Z. C., et al. (2017b). Synthesis, characterization and frictional wear behavior of ceria hybrid architectures with $\{111\}$ exposure planes. Appl. Surf. Sci. 401, 100-105. doi: 10.1016/j.apsusc.2017.01.005

Parthibavarman, M., Karthik, M., and Prabhakaran, S. (2018). Facile and one step synthesis of $\mathrm{WO}_{3}$ nanorods and nanosheets as an efficient photocatalyst and humidity sensing material. Vacuum 155, 224-232. doi: 10.1016/j.vacuum.2018.06.021

Sun, R., Wang, Z. C., Saito, M., Shibata, N., and Ikuhara, Y. (2015). Atomistic mechanisms of nonstoichiometry-induced twin boundary structural transformation in titanium dioxide. Nat. Commun. 6:7120. doi: $10.1038 /$ ncomms 8120

Wang, Z. C., Saito, M., McKenna, K. P., Fukami, S., Sato, H., Ikeda, S., et al. (2016). Atomic-scale structure and local chemistry of CoFeB-MgO magnetic tunnel junctions. Nano Lett. 16, 1530-1536. doi: 10.1021/acs.nanolett.5b03627
Wang, Z. C., Saito, M., McKenna, K. P., Gu, L., Tsukimoto, S., Shluger, A. L., et al. (2011). Atom-resolved imaging of ordered defect superstructures at individual grain boundaries. Nature 479, 380-383. doi: 10.1038/nature10593

Wang, Z. C., Saito, M., McKenna, K. P., and Ikuhara, Y. (2014). Polymorphism of dislocation core structures at the atomic scale. Nate Commun. 5:3239 doi: $10.1038 /$ ncomms4239

Xiao, J., Song, C. W., Dong, W., Chen, L., and Yanyan, Y. (2017). Synthesis, characterization, and gas sensing properties of $\mathrm{WO}_{3}$ nanoplates. Rare Metal Mater. Eng. 46, 1241-1244. doi: 10.1016/S1875-5372(17)30144-3

Xu, L., Gu, D. X., Chang, X. T., Chai, L. G., Li, Z., Jin, X., et al. (2017). Rareearth-doped tungsten oxide microspheres with highly enhanced photocatalytic activites. Ceram. Int. 43, 10263-10269. doi: 10.1016/j.ceramint.2017.05.055

Zhan,Y., Liu, Y. L., Liu, Q. Q., Liu, Z. M., Yang, H., Lei, B., et al. (2018). Sizecontrolled synthesis of fluorescent tungsten oxide quantum dots via one-pot ethanol-thermal strategy for ferric ions detection and bioimaging. Sens. Actuats B Chem. 255, 290-298. doi: 10.1016/j.snb.2017.08.043

Zhang, J., Fu, X., Hao, H., and Gan, W. (2018). Facile synthesis 3D flowerlike $\mathrm{Ag} @ \mathrm{WO}_{3}$ nanostructures and applications in solar-light photocatalysis. J. Alloys Compd. 757,134-141. doi: 10.1016/j.jallcom.2018.05.068

Conflict of Interest Statement: The authors declare that the research was conducted in the absence of any commercial or financial relationships that could be construed as a potential conflict of interest.

Copyright (c) $2018 \mathrm{Hu}, \mathrm{Hu}$, Chen, Lin and Qiu. This is an open-access article distributed under the terms of the Creative Commons Attribution License (CC BY). The use, distribution or reproduction in other forums is permitted, provided the original author(s) and the copyright owner(s) are credited and that the original publication in this journal is cited, in accordance with accepted academic practice. No use, distribution or reproduction is permitted which does not comply with these terms. 